\title{
On Fuzzy Differential Inequalities with Upper and Lower Solutions
}

\author{
Ali Yakar $^{1}$ (D) , Seda Çağlak ${ }^{2}$ (I) \\ Article History \\ Received: 08 Dec 2021 \\ Accepted: 23 Dec 2021 \\ Published: 31 Dec 2021 \\ 10.53570/jnt.1034452 \\ Research Article \\ Abstract - In this article, by using the technique of upper and lower solutions, \\ some comparison results for first-order fuzzy differential equations are established. \\ Hukuhara derivative, Hukuhara difference, and partial orderings are used for proving \\ theorems. We extend our results to initial time difference case as well. Also, the \\ practicality of these comparison results is demonstrated by giving an example.
}

Keywords - Fuzzy number, upper and lower solutions, Hukuhara derivative

Mathematics Subject Classification (2020) - 28E10, 26D10

\section{Introduction}

Fuzzy mathematics is an interesting part of mathematics. It could be said a new branch of mathematics since it emerged in the 1960s. It was proposed to explain real-life in mathematical language. We use relative concepts a lot in our daily lives. Situations that can vary from person to person such as good and bad, young and old, beautiful and ugly, had taken place in our lives. Classical mathematics is not sufficient to explain such notions. Everything is certain in classical mathematics, something is either black or white. Classical mathematics does not allow gray color. At this point where classical mathematics is insufficient, Lutfi Askerzade Zadeh's fuzzy mathematical theory comes into play. He showed in his theory that he revealed in 1965, fuzzy logic constitutes a good model of real-life [1]. Although his theory was not accepted in the scientific community at first, it is now used in different branches of science and engineering.

The fuzzy theory attracted great attention and spread very commonly among scientists from various fields of science. Fuzzy systems can also be used in predicting, decision making, air conditioning, mechanical control systems such as automobile control systems and intelligent structures, as well as industrial process control systems, and so on. See [2-4] and the references cited therein.

In fuzzy mathematics, arithmetical operations are valid like in classical mathematics. There are two methods. One is Zadeh Extension Principle and the other is interval arithmetic with level sets. These methods are explained in [5]. There are some differences between fuzzy mathematics and classical mathematics. For example, if $u$ is a fuzzy number, $u-u$ may not be 0 in fuzzy mathematics. As a result of this issue, a new difference known as the Hukuhara difference is defined, as shown in [6]. By using Hukuhara difference, Hukuhara derivative is defined [7]. On the other hand, it is possible to compare two fuzzy numbers or two fuzzy functions. How to compare two fuzzy notions are mentioned in [8]. Besides, it is possible solving fuzzy differential equations. Many methods were developed to solve fuzzy differential equations. How to solve first-order fuzzy differential equations are explained

\footnotetext{
${ }^{1}$ ali.yakar@gop.edu.tr; ${ }^{2}$ caglakseda@gmail.com (Corresponding Author)

${ }^{1,2}$ Department of Mathematics, Tokat Gaziosmanpaşa University, Tokat, Turkey
} 
in [5]. Besides this, the definition of fuzzy upper and lower solutions are located in [9]. In this study, by using all the knowledge mentioned above, some comparison results are proved and one example is introduced to support the results.

\section{Preliminaries}

Hukuhara difference is a useful operation for the difference between fuzzy notions and also, interval arithmetic via level sets is an easy method for arithmetical operations in fuzzy cases. In the following, definitions of fuzzy numbers, level sets of a fuzzy number, Hukuhara difference, and Hukuhara derivative are given.

Definition 2.1. [5] A fuzzy set $U$ (fuzzy subset of $E$ ) is defined as a mapping ( $E$ is universal set)

$$
\mu_{u}: E \rightarrow[0,1]
$$

where $\mu_{u}(x)$ is the membership degree of $x$ the fuzzy set $U$.

Definition 2.2. [10] A fuzzy membership function $u: \mathbb{R} \rightarrow[0,1]$ is called a fuzzy number if it has the following conditions:

i. $u$ is normal. It means there is at least a real member $x_{0} \in \mathbb{R}$ such that $u\left(x_{0}\right)=1$.

ii. $u$ is fuzzy convex. It means for two arbitrary real points $x_{1}, x_{2} \in \mathbb{R}$ and $t \in[0,1]$, we have

$$
u\left(t x_{1}+(1-t) x_{2}\right) \geq \min \left\{u\left(x_{1}\right), u\left(x_{2}\right)\right\}
$$

iii. $u$ is upper semi-continuous on $\mathbb{R}$. It means if we increase its value at a certain point $x_{0}$ to $f\left(x_{0}\right)+\varepsilon$ (for some positive constant $\varepsilon$ ), then the result is upper-semicontinuous; if we decrease its value to $f\left(x_{0}\right)-\varepsilon$ then the result is lower-semi-continuous.

iv. $u$ is compactly supported. It means the closure of the set $\{x \in \mathbb{R} ; u(x)>0\}$, as a support set, is compact set.

Remark 2.3. The space of fuzzy numbers will be denoted by $\mathbb{R}_{\mathcal{F}}$.

Definition 2.4. Let $U: E \rightarrow[0,1]$ be a fuzzy set. The level sets of $U$ are defined as the classical sets.

$$
U_{r}=\left[u_{r}^{-}, u_{r}^{+}\right]=\left\{x \in E: \mu_{U}(x) \geq r\right\}, \quad 0<r<1
$$

Remark 2.5. There are numerous fuzzy numbers. However, in our investigation, we only mention triangular and trapezoidal numbers. As a result, we define these fuzzy numbers as follows:

A trapezoidal fuzzy number $u$ can be shown with an ordered pair of functions $(a, b, c, d) \in \mathbb{R}^{4}$,

$$
u(x)= \begin{cases}0, & t<a \\ \frac{t-a}{b-a}, & a \leq t \leq b \\ 1, & b \leq t \leq c \\ \frac{d-t}{d-c}, & c<t \leq d \\ 0, & d<t\end{cases}
$$

Employing $r$-cut approach, we find the level set with the endpoints calculated by

$$
u_{r}^{-}=a+r(b-a), u_{r}^{+}=d-r(d-c)
$$

where $r \in[0,1]$. When $b=c$ is in the form (a,b,c,d), the fuzzy number is referred to as a triangular fuzzy number. The fuzzy number may thus be represented by a triplet $(a, b, c) \in \mathbb{R}^{3}, a \leq b \leq c$. 
Definition 2.6. [6] The Hukuhara difference (H-difference $\ominus_{H}$ ) defined by

$$
u \ominus_{H} v=w \Leftrightarrow u=v+w
$$

being + the standard fuzzy addition.

If $u \ominus_{H} v$ exists, its level-sets are

$$
\left[u \ominus_{H} v\right]_{r}=\left[u_{r}^{-}-v_{r}^{-}, u_{r}^{+}-v_{r}^{+}\right]
$$

Definition 2.7. [11] A function $\psi:(a, b) \rightarrow \mathbb{R}_{\mathcal{F}}$ is called Hukuhara differentiable if for sufficiently small $h>0$ the $H$-differences $\psi(u+h) \ominus \psi(u)$ and $\psi(u) \ominus \psi(u-h)$ exist and if there exists an element $\psi^{\prime}(u) \in \mathbb{R}_{\mathcal{F}}$ such that

$$
\lim _{h \rightarrow 0} \frac{\psi(u+h) \ominus \psi(u)}{h}=\lim _{h \rightarrow 0} \frac{\psi(u) \ominus \psi(u-h)}{h}=\psi^{\prime}(u)
$$

The fuzzy number $\psi(u)$ is called the Hukuhara derivative of $\psi$ at $u$.

Lemma 2.8. Let $u$ be Hukuhara differentiable and $u(t)=(x(t), y(t), z(t)))$ be triangular number valued function then we get $u^{\prime}=\left(x^{\prime}, y^{\prime}, z^{\prime}\right)$.

Definition 2.9. [8] Let $u, v \in \mathbb{R}_{\mathcal{F}}$. It is said $u \leq v$ if only if $u_{r}^{-} \leq v_{r}^{-}$and $u_{r}^{+} \leq v_{r}^{+}$for each $r \in[0,1]$.

Definition 2.10. [8] Let $u, v \in \mathbb{R}_{\mathcal{F}}$. It is said $u \preceq v$ if only if $u_{r}^{-} \geq v_{r}^{-}$and $u_{r}^{+} \leq v_{r}^{+}$for each $r \in[0,1]$, that is $u_{r} \subseteq v_{r}, \forall r \in[0,1]$.

Lemma 2.11. [8] If $u, v, w \in \mathbb{R}_{\mathcal{F}}$ are such that Hukuhara differences $u \ominus_{H} w$ and $v \ominus_{H} w$ exist, then

$$
u \leq v \Leftrightarrow u \ominus_{H} w \leq v \ominus_{H} w
$$

Definition 2.12. [9] Let $\Psi \in C\left(\left[t_{0}, T\right] \times \mathbb{R}_{\mathcal{F}}, \mathbb{R}_{\mathcal{F}}\right), T>t_{0}$ and $x^{\prime}=\Psi(t, x), x\left(t_{0}\right)=x_{0}$ be fuzzy initial value problem.

A function $q \in C^{1}\left(\left[t_{0}, T\right], \mathbb{R}_{\mathcal{F}}\right)$ is an upper solution for fuzzy initial value problem above, if

$$
\left\{\begin{array}{l}
q^{\prime}(t) \geq \Psi(t, q(t)), \quad t \in\left[t_{0}, T\right] \\
q\left(t_{0}\right) \geq x_{0}
\end{array}\right.
$$

A function $p \in C^{1}\left(\left[t_{0}, T\right], \mathbb{R}_{\mathcal{F}}\right)$ is a lower solution for fuzzy initial value problem above, if

$$
\left\{\begin{array}{l}
p^{\prime}(t) \leq \Psi(t, p(t)), \quad t \in\left[t_{0}, T\right] \\
p\left(t_{0}\right) \leq x_{0}
\end{array}\right.
$$

Remark 2.13. Analogous definitions can be given for $\preceq$ partial ordering.

\section{Main Results}

In this section, several comparison results are given by using upper and lower solutions and, it is also proved that the solution of fuzzy differential equation exists between corresponding lower and upper solutions.

Let us consider the following first order fuzzy differential equation

$$
x^{\prime}=\Psi(t, x), x\left(t_{0}\right)=x_{0}
$$

where $\Psi \in C\left(\left[t_{0}, T\right] \times \mathbb{R}_{\mathcal{F}}, \mathbb{R}_{\mathcal{F}}\right)$ and $T>t_{0}$. 
Theorem 3.1. Let the functions $p, q \in C^{1}\left(\left[t_{0}, T\right], \mathbb{R}_{\mathcal{F}}\right)$ satisfy the following inequalities

$$
q^{\prime}>\Psi(t, q(t)), \quad p^{\prime} \leq \Psi(t, p(t))
$$

If $p\left(t_{0}\right)<q\left(t_{0}\right)$, then $p(t)<q(t)$ for all $t \in\left[t_{0}, T\right]$.

Proof. Since we are going to prove $p(t)<q(t)$ by Definition 2.9, we must show that $p_{r}^{-}(t)<q_{r}^{-}(t)$ and $p_{r}^{+}<q_{r}^{+}$on $t \in\left[t_{0}, T\right]$. If it was wrong, there would exists a $t_{1} \in\left(t_{0}, T\right]$ such that

$$
p_{r}^{-}\left(t_{1}\right)=q_{r}^{-}\left(t_{1}\right), p_{r}^{+}\left(t_{1}\right)=q_{r}^{+}\left(t_{1}\right)
$$

and

$$
p_{r}^{-}(t)<q_{r}^{-}(t), p_{r}^{+}(t)<q_{r}^{+}(t)
$$

for $t \in\left[t_{0}, t_{1}\right)$. Therefore, for sufficiently small $h>0$, we obtain

$$
\left\{\begin{array}{l}
p_{r}^{-}\left(t_{1}-h\right)<q_{r}^{-}\left(t_{1}-h\right) \\
p_{r}^{+}\left(t_{1}-h\right)<q_{r}^{+}\left(t_{1}-h\right)
\end{array}\right.
$$

i.e. $p\left(t_{1}-h\right)<q\left(t_{1}-h\right)$. By using Lemma 2.11 , we have

$$
p\left(t_{1}-h\right) \ominus p\left(t_{1}\right)<q\left(t_{1}-h\right) \ominus q\left(t_{1}\right)
$$

By dividing inequality with $-h$ and limiting for $h \rightarrow 0$, we arrive at

$$
\lim _{h \rightarrow 0} \frac{p\left(t_{1}-h\right) \ominus p\left(t_{1}\right)}{-h} \geq \lim _{h \rightarrow 0} \frac{q\left(t_{1}-h\right) \ominus q\left(t_{1}\right)}{-h}
$$

which implies that

$$
p^{\prime}\left(t_{1}\right) \geq q^{\prime}\left(t_{1}\right)
$$

As a result, we get a contradiction

$$
\Psi\left(t_{1}, p\left(t_{1}\right)\right) \geq p^{\prime}\left(t_{1}\right) \geq q^{\prime}\left(t_{1}\right)>\Psi\left(t_{1}, q\left(t_{1}\right)\right)=\Psi\left(t_{1}, p\left(t_{1}\right)\right)
$$

So we conclude that $p(t)<q(t)$ on $\left[t_{0}, T\right]$.

Similarly it can be shown that the result of the theorem holds even when we change $\leq$ by $<$ and $\geq$ by $>$ in the assumptions of theorem 3.1 .

We are in position to show that the existence of the solution of the problem (1) located between corresponding lower and upper solutions.

Theorem 3.2. Let the functions $p, q \in C^{1}\left(\left[t_{0}, T\right], \mathbb{R}_{\mathcal{F}}\right)$ satisfy the strict inequalities $p^{\prime}<\Psi(t, p)$ and $q^{\prime}>\Psi(t, q)$ on $\left[t_{0}, T\right]$. Furthermore, $y$ is the solution of the problem $(1)$. Then, $p\left(t_{0}\right) \leq y\left(t_{0}\right) \leq q\left(t_{0}\right)$ implies that $p(t)<y(t)<q(t)$ for $\left[t_{0}, T\right]$.

Proof. We shall only give a proof for $y(t)<q(t)$ on $\left[t_{0}, T\right]$. If $y\left(t_{0}\right)<q\left(t_{0}\right)$, then the result follows from Theorem 3.1. We now suppose that $y\left(t_{0}\right)=q\left(t_{0}\right)$ and, we define the function $z$ such as $z(t)=q(t) \ominus y(t)$ on $\left[t_{0}, T\right]$. Then, the level sets of this function can be written as $Z_{r}=\left[z_{r}^{-}, z_{r}^{+}\right]$, that is,

$$
\left\{\begin{array}{l}
z_{r}^{-}=q_{r}^{-}-y_{r}^{-} \\
z_{r}^{+}=q_{r}^{+}-y_{r}^{+}
\end{array}\right.
$$

If we differentiate the both sides of the real function $z_{r}^{-}$, we find that

$$
\left(z_{r}^{-}\right)^{\prime}\left(t_{0}\right)=\left(q_{r}^{-}\right)^{\prime}\left(t_{0}\right)-\left(y_{r}^{-}\right)^{\prime}\left(t_{0}\right)>\Psi\left(t_{0},\left(q_{r}^{-}\right)^{\prime}\left(t_{0}\right)\right)-\Psi\left(t_{0},\left(y_{r}^{-}\right)^{\prime}\left(t_{0}\right)\right)=0
$$

It is easy to observe that $z_{r}^{-}(t)$ is increasing on the interval $\left[t_{0}, t_{0}+h\right]$ for sufficiently small $h>0$. For this reason, we obtain

$$
y_{r}^{-}\left(t_{0}+h\right)<q_{r}^{-}\left(t_{0}+h\right)
$$


If we utilize Theorem 3.1, we can easily see that $y_{r}^{-}(t)<q_{r}^{-}(t)$, for all $t \in\left[t_{0}+h, T\right]$. Analogously,

$$
\begin{aligned}
\left(z_{r}^{+}\right)^{\prime}\left(t_{0}\right) & =\left(q_{r}^{+}\right)^{\prime}\left(t_{0}\right)-\left(y_{r}^{+}\right)^{\prime}\left(t_{0}\right) \\
& >\Psi\left(t_{0},\left(q_{r}^{+}\right)^{\prime}\left(t_{0}\right)\right)-\Psi\left(t_{0},\left(y_{r}^{+}\right)^{\prime}\left(t_{0}\right)\right) \\
& =0
\end{aligned}
$$

We can easily see that $z_{r}^{+}$is increasing to the right of $t_{0}$ in a sufficiently small interval $\left[t_{0}, t_{0}+h\right]$. Hence,

$$
y_{r}^{+}\left(t_{0}+h\right)<q_{r}^{+}\left(t_{0}+h\right), y_{r}^{-}(t)<q_{r}^{-}(t)
$$

for all $t \in\left[t_{0}+h, T\right]$. Since we have chosen sufficiently small $h$, we obtain $y(t)<q(t)$ on $\left[t_{0}, T\right]$ due to

$$
\left\{\begin{array}{l}
y_{r}^{-}(t)<q_{r}^{-}(t) \\
y_{r}^{+}(t)<q_{r}^{+}(t)
\end{array}\right.
$$

In a similar way, we can prove $p(t)<y(t)$ on $\left[t_{0}, T\right]$. Thus, we have

$$
p(t)<y(t)<q(t) \text { on }\left[t_{0}, T\right]
$$

Theorem 3.3. Let $\Psi \in C\left(\left[t_{0}, T\right] \times \mathbb{R}_{\mathcal{F}}, \mathbb{R}_{\mathcal{F}}\right)$ and the functions $p, q \in C^{1}\left(\left[t_{0}, T\right], \mathbb{R}_{\mathcal{F}}\right)$ be lower and upper solutions of (1) respectively. Suppose that Hukuhara differences $\Psi(t, x) \ominus \Psi(t, y)$ and $x \ominus y$ exist and for $x \geq y$, there exist a positive constant $L$ such that

$$
\Psi(t, x) \ominus \Psi(t, y) \leq L(x \ominus y)
$$

Then, $p(t) \leq q(t)$ on $\left[t_{0}, T\right]$ provided that $p\left(t_{0}\right) \leq q\left(t_{0}\right)$.

Proof. Firstly, we define the function $\tilde{q}(t)=q(t)+\varepsilon \cdot e^{2 L t}$ for some $\varepsilon>0$. Let the level sets be $\tilde{q}_{r}=\left[\tilde{q}_{r}^{-}(t), \tilde{q}_{r}^{+}(t)\right]$, i.e.

$$
\left\{\begin{array}{l}
\tilde{q}_{r}^{-}(t)=q_{r}^{-}(t)+\varepsilon \cdot e^{2 L t} \\
\tilde{q}_{r}^{+}(t)=q_{r}^{+}(t)+\varepsilon \cdot e^{2 L t}
\end{array}\right.
$$

Observe that $p_{r}^{-}\left(t_{0}\right) \leq q_{r}^{-}\left(t_{0}\right)<\tilde{q}_{r}^{-}\left(t_{0}\right)$. Now, taking derivatives of both sides of $q_{r}^{-}(t)$, we get

$$
\begin{aligned}
\frac{d}{d t}\left(\tilde{q}_{r}^{-}(t)\right) & =\frac{d}{d t}\left(q_{r}^{-}(t)\right)+2 L \varepsilon \cdot e^{2 L t} \\
& \geq \Psi\left(t, q_{r}^{-}(t)\right)+2 L \varepsilon e^{2 L t} \\
& \geq \Psi\left(t, q_{r}^{-}(t)\right)-\Psi\left(t, \tilde{q}_{r}^{-}(t)\right)+2 L \varepsilon e^{2 L t}+\Psi\left(t, \tilde{q}_{r}^{-}(t)\right)
\end{aligned}
$$

By using one sided Lipschitz type inequality (3.3), it follows

$$
\frac{d}{d t}\left(\tilde{q}_{r}^{-}(t)\right) \geq-L\left(\tilde{q}_{r}^{-}(t)-q_{r}^{-}(t)\right)+\Psi\left(t, \tilde{q}_{r}^{-}(t)\right)+L \varepsilon e^{2 L t}>\Psi\left(t, \tilde{q}_{r}^{-}(t)\right)
$$

An application of Theorem 3.1 gives that $p_{r}^{-}(t)<\tilde{q}_{r}^{-}(t)$. Letting $\varepsilon \rightarrow 0$, we obtain $p_{r}^{-}(t) \leq q_{r}^{-}(t)$ on $\left[t_{0}, T\right]$. Analogously,

$$
\begin{aligned}
\frac{d}{d t}\left(\tilde{q}_{r}^{+}(t)\right) & =\frac{d}{d t}\left(q_{r}^{+}(t)\right)+2 L \varepsilon e^{2 L t} \\
& \geq \Psi\left(t, q_{r}^{+}(t)\right)+2 L \varepsilon e^{2 L t} \\
& \geq \Psi\left(t, q_{r}^{+}(t)\right)-\Psi\left(t, \tilde{q}_{r}^{+}(t)\right)+2 L \varepsilon e^{2 L t}+\Psi\left(t, \tilde{q}_{r}^{+}(t)\right) \\
& \geq-L\left(\tilde{q}_{r}^{+}(t)-q_{r}^{+}(t)\right)+\Psi\left(t, \tilde{q}_{r}^{+}(t)\right)+L \varepsilon e^{2 L t} \\
& >\Psi\left(t, \tilde{q}_{r}^{+}(t)\right)
\end{aligned}
$$

Also by Theorem 3.1 and the fact that $p_{r}^{+}\left(t_{0}\right) \leq q_{r}^{+}\left(t_{0}\right)<\tilde{q}_{r}^{+}\left(t_{0}\right)$, we conclude that $p_{r}^{+}(t)<\tilde{q}_{r}^{+}(t)$, meaning that $p_{r}^{+}(t) \leq q_{r}^{+}(t)$ when $\varepsilon$ goes to zero. According to Definition 2.9, we deduce that $p(t) \leq$ $q(t)$ on $\left[t_{0}, T\right]$ which completes the proof. 
Remark 3.4. The same conclusions are valid for $\preceq$ partial ordering

Example 3.5. Consider fuzzy initial value problem $x^{\prime}=x, x(0)=(2,3,4)$ where $\Psi(t, x)=x$. We consider the functions $p(t)=\left(-t^{2}+1\right)(0,1,2)$ and $w(t)=e^{t}(5,6,7)$ for $t \in[0,1]$. Let us try to examine that whether the inequality $p^{\prime}(t) \leq \Psi(t, p)$ holds for $t \in[0,1]$. Here, $p^{\prime}(t)=-2 t(0,1,2)$ and $\Psi(t, p)=\left(-t^{2}+1\right)(0,1,2)$. Since $p^{\prime}(t)=(0,-2 t,-4 t)$ then by Lemmma 2.8 and Remark 2.5 , the level sets can be written as

$$
\left[p^{\prime}(t)\right]_{r}=\left[\left(p^{\prime}(t)\right)_{r}^{-},\left(p^{\prime}(t)\right)_{r}^{+}\right]=[-2 t r,-2 t(2+r)]
$$

and we also have $\Psi(t, p)=\left(0,-t^{2}+1,-2 t^{2}+2\right)$. On the other hand, we acquire

$$
\left.[\Psi(t, p)]_{r}=\left[(\Psi(t, p))_{r}^{-}\right],(\Psi(t, p))_{r}^{+}\right]=\left[\left(-t^{2}+1\right) r,\left(-t^{2}+1\right)(2-r)\right]
$$

It is easily observe that

$$
\left(p^{\prime}(t)\right)_{r}^{-} \leq(\Psi(t, p))_{r}^{-} \quad \text { and } \quad\left(p^{\prime}(t)\right)_{r}^{+} \leq(\Psi(t, p))_{r}^{+}
$$

for $r \in[0,1]$, i.e., $p^{\prime} \leq \Psi(t, p)$ on $[0,1]$. Now, looking for that whether $p(0) \leq x(0)$ is true. Since $p(0)=$ $(0,1,2)$, its level set $\left.[p(0)]_{r}\right]=[r, 2-r]$ and since $x(0)=(2,3,4)$, its level sets $[x(0)]_{r}=[2+r, 4-r]$. Therefore, we can see that

$$
\left((p(0))_{r}^{-} \leq\left(( x ( 0 ) ) _ { r } ^ { - } \quad \text { and } \quad \left((p(0))_{r}^{+} \leq\left((x(0))_{r}^{+}\right.\right.\right.\right.
$$

So, $p(0) \leq x(0)$. Finally, $p$ is the lower solution of fuzzy differential equation. Like mentioned above, if we take $q(t)=e^{t}(5,6,7)$ and $\Psi(t, q)=e^{t}(5,6,7)$, then we can similarly show that

$$
q^{\prime}(t) \geq \Psi(t, q), q(0) \geq x(0)
$$

Consequently, $q$ is the upper solution of the given fuzzy differential equation. It is obvious that the fuzzy differential equation satisfies the one sided Lipschitz condition given in (3.3) for $L>1$. Moreover, by comparing the following level sets that we have just found

$$
[p(0)]_{r}=[r, 2-r] \quad \text { and } \quad[q(0)]_{r}=[5+r, 7-r]
$$

We see that

$$
[p(0)]_{r}^{-} \leq[q(0)]_{r}^{-} \quad \text { and } \quad[p(0)]_{r}^{+} \leq[q(0)]_{r}^{+}
$$

for $r \in[0,1]$, so the inequality $p(0) \leq q(0)$ holds. As a consequence, we obtain $p(t) \leq q(t)$ on $[0,1]$ by Theorem 3.3. Actually, we can check directly whether the result holds. To do so, let us construct the level sets of $p(t)$ and $q(t)$ :

$$
[p(t)]_{r}=\left[\left(-t^{2}+1\right) r,\left(-t^{2}+1\right)(2-r)\right] \quad \text { and } \quad[q(t)]_{r}=\left[e^{t}(5+r), e^{t}(7-r)\right]
$$

and it can be easily seen that

$$
[p(t)]_{r}^{-} \leq[q(t)]_{r}^{-} \quad \text { and } \quad[p(t)]_{r}^{+} \leq[q(t)]_{r}^{+}
$$

for all $r \in[0,1]$. Thus, $p(t) \leq q(t)$ on $[0,1]$ is obtained. Furthermore, one can get the exact solution of the problem as follows:

$$
y=e^{t}(2,3,4)
$$

Observe that, $p(t) \leq y(t) \leq q(t), t \in[0,1]$, it's represented in Figure 1 . 


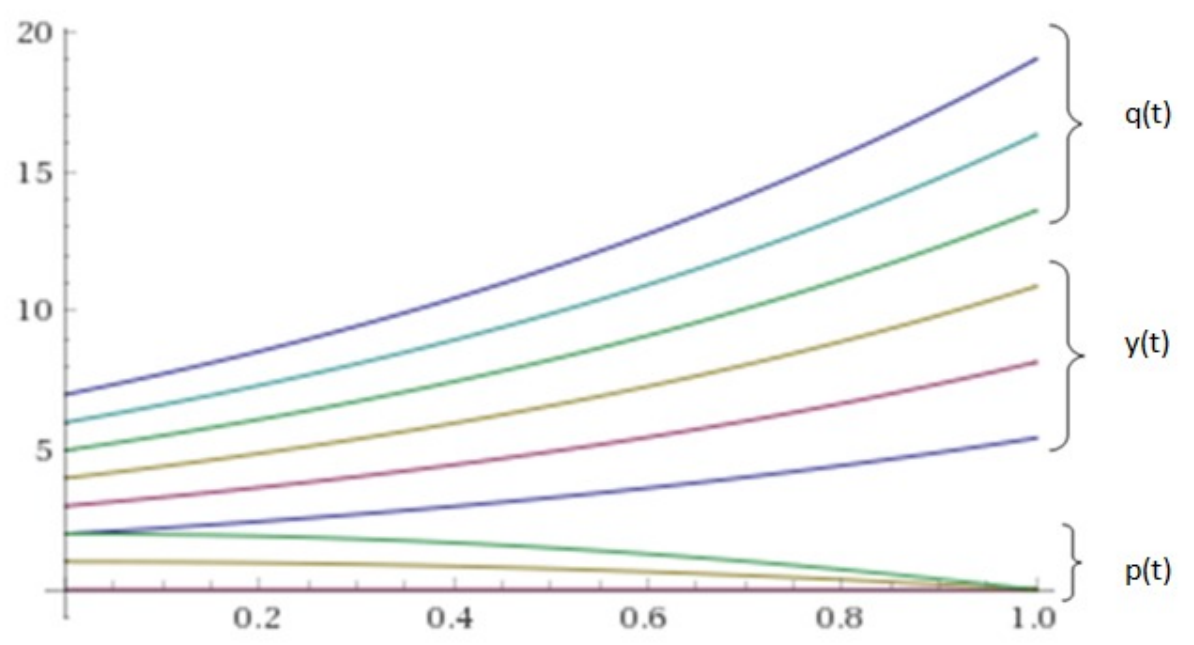

Fig. 1

In some cases, lower and upper solutions may differ at initial points. In such instances, we need to make some changes to the previous theorem and add extra conditions to achieve the similar results.

Theorem 3.6. Assume that

(H1) $\Psi \in C\left(\left[t_{0}, \tau_{0}+T\right] \times \mathbb{R}_{\mathcal{F}}, \mathbb{R}_{\mathcal{F}}\right), \tau_{0} \geq t_{0}, T>0$ and the functions $p \in C^{1}\left(\left[t_{0}, t_{0}+T\right], \mathbb{R}_{\mathcal{F}}\right)$, $q \in C^{1}\left(\left[\tau_{0}, \tau_{0}+T\right], \mathbb{R}_{\mathcal{F}}\right)$ such that

$$
\begin{aligned}
& p^{\prime} \leq \Psi(t, p), p\left(t_{0}\right) \leq x_{0}, \\
& q^{\prime} \geq \Psi(t, q), q\left(\tau_{0}\right) \geq x_{0},
\end{aligned}
$$

(H2) $\Psi(t, x) \ominus \Psi(t, y) \leq L(x \ominus y), x \geq y, L>0$

(H3) $\Psi(t, x)$ is non decreasing in $t$ for each $x$.

Then we have

(i) $p(t) \leq q(t+\gamma)$ on $\left[t_{0}, t_{0}+T\right]$ or

(ii) $p(t-\gamma) \leq q(t)$ on $\left[\tau_{0}, \tau_{0}+T\right]$

where $\gamma=\tau_{0}-t_{0}$.

\section{Proof.}

(i) Define the function $q_{0}(t)=q(t+\gamma)$ thus we get

$$
q_{0}\left(t_{0}\right)=q\left(t_{0}+\gamma\right)=q\left(\tau_{0}\right) \geq x_{0} \geq p\left(t_{0}\right)
$$

and

$$
q^{\prime}(t)=q^{\prime}(t+\gamma) \geq \Psi(t+\gamma, q(t+\gamma))=\Psi\left((t+\gamma), q_{0}(t)\right)>\Psi\left(t, q_{0}(t)\right)
$$

So, $q$ is an upper solution. By using Theorem 3.3 the proof is completed.

(ii) Define the function $p_{0}(t)=p(t-\gamma), t \geq \tau_{0}$. It can be written as before

$$
p_{0}\left(\tau_{0}\right)=p_{0}\left(\tau_{0}-\gamma\right)=p_{0}\left(t_{0}\right) \leq x_{0} \leq q\left(\tau_{0}\right)
$$

and

$$
p_{0}^{\prime}(t)=p^{\prime}(t-\gamma) \leq \Psi(t-\gamma, p(t-\gamma))=\Psi\left(t-\gamma, p_{0}(t)\right)
$$

which leads to the fact $p$ is a lower solution. As a result, by using the previous Theorem, (ii) is proved. 


\section{Conclusion}

In this paper, it is aimed to prove some comparison theorems by utilizing upper and lower solutions for fuzzy differential equations of first order. Our results have been refined to initial time difference case where upper and lower solutions start different points in time. In addition, an example has been given for illustration.

\section{Author Contributions}

All the authors contributed equally to this work. They all read and approved the final version of the manuscript.

\section{Conflicts of Interest}

The authors declare no conflict of interest.

\section{References}

[1] L. A. Zadeh, Fuzzy sets, World Scientific, 1996.

[2] C.-W. Chen, Stability Conditions of Fuzzy Systems and Its Application to Structural and Mechanical Systems, Advances in Engineering Software 37 (9) (2006) 624-629.

[3] Q. Shen, B. Jiang, V. Cocquempot, Fault-Tolerant Control for T-S Fuzzy Systems with Application to Near-Space Hypersonic Vehicle with Actuator Faults, IEEE Transactions on Fuzzy Systems 20 (4) (2011) 652-665.

[4] T. Terano, K. Asai, M. Sugeno, Fuzzy Systems Theory and Its Applications, Academic Press Professional, Inc., 1992.

[5] B. Bede, Fuzzy Sets, Springer, 2013.

[6] M. Hukuhara, Integration des Applications Mesurables dont la Valeur est un Compact Convexe, Funkcialaj Ekvacioj 10 (3) (1967) 205-223.

[7] P. Diamond, P. Kloeden, Metrics of Fuzzy Sets: Theory and Applications, World Scientific, 1994.

[8] R. Rodríguez-López, Comparison Results for Fuzzy Differential Equations, Information Sciences 178 (6) (2008) 1756-1779.

[9] R. Rodríguez-López, Monotone Method for Fuzzy Differential Equations, Fuzzy Sets and Systems 159 (16) (2008) 2047-2076.

[10] T. Allahviranloo, Fuzzy Fractional Differential Operators and Equations, Vol. 397, Springer, 2020.

[11] M. L. Puri, D. A. Ralescu, Differentials of Fuzzy Functions, De Gruyter Mouton, 2011. 